\title{
The Impact of Comorbidities on Calgary Hospital Utilization in Patients With Chronic Obstructive Pulmonary Disease and Heart Failure
}

Douglas C. Woodhouse ${ }^{1}$, Alexandra D. Frolkis ${ }^{2}$, Brenna J. Murray ${ }^{3}$, Nathan M. Solbak ${ }^{3}$, Najla Samardzic ${ }^{3}$ , Kelly W. Burak ${ }^{2}$

1. Family Medicine, University of Calgary, Calgary, CAN 2. Internal Medicine, University of Calgary, Calgary, CAN 3. Medicine, University of Calgary, Calgary, CAN

Corresponding author: Douglas C. Woodhouse, douglas.woodhouse@ucalgary.ca

\section{Abstract}

\section{Background}

Chronic obstructive pulmonary disease (COPD) and heart failure (HF) are chronic conditions with high acute care utilization. Disease-specific order sets were developed for patients with COPD or HF in Calgary to reduce total days in hospital for this population of patients. However, many patients have comorbidities which may contribute to hospital utilization; thus, disease-specific order sets may not be an optimal solution to reduce overall acute care utilization.

\section{Methods}

Inpatient data on Calgary hospitalizations for COPD or HF between April 1, 2017 - March 31, 2019 and associated diagnoses were identified. Outcomes included total days in hospital and length of stay for COPD and HF patients stratified by number of comorbidities.

\section{Results}

Total days in hospital increased with the number of comorbidities for both conditions. During the study period, 131 patients with COPD and no comorbidities had a median length of stay of three days (IQR: 3) compared to 3,911 COPD patients with one to five comorbidities with a median length of stay of seven days (IQR: 9). There were 47 patients with HF and no comorbidities with a median length of stay of four days (IQR: 5) compared to 6,273 HF patients with one to five comorbidities with a median length of stay of nine days (IQR: 12). Common comorbidities included hypertension, type 2 diabetes, and acute renal failure. COPD and HF are frequently comorbid.

\section{Conclusions}

Review began 07/15/2021 Review ended 08/11/2021 Published 08/19/2021

\section{(๑) Copyright 2021}

Woodhouse et al. This is an open access article distributed under the terms of the Creative Commons Attribution License CC-BY 4.0., which permits unrestricted use, distribution, and reproduction in any medium, provided the original author and source are credited.
Total days in hospital for patients with COPD or HF is positively correlated with the number of comorbidities. COPD or HF patients with between one to five comorbidities (compared to those with no comorbidities, and those with more than five comorbidities) represent the majority of total days in hospital, and the majority of patients. This highlights the importance of focusing on patients with comorbidities in efforts to reduce hospital utilization, and suggests that concurrent management of commonly occurring comorbidities for HF and COPD patients may be necessary to achieve this goal.

Categories: Pulmonology, Quality Improvement

Keywords: copd, heart failure, length of hospital stay (los), hospitalization, medical comorbidities

\section{Introduction}

Chronic obstructive pulmonary disease (COPD) and heart failure (HF) are chronic conditions with high morbidity and acute care utilization. They are among the top four diagnoses for inpatient hospitalizations in Alberta [1]. Many guidelines have been developed to encourage wider adoption of evidence-based practices for the management of patients with these conditions [2,3]. An estimated \$1 billion was spent from 2008 to 2016 in Alberta for COPD care, with the majority of costs associated with hospitalization [4]. Evidencebased, disease-specific acute care order sets for COPD and HF are being implemented in Calgary hospitals, with an intention to spread provincially and to community care [5]. The primary goal is to reduce total days in hospital for patients with either of these conditions. However, the order sets are not designed to optimize the coordinated management of commonly occurring co-morbidities, potentially limiting their clinical utility.

At least one comorbidity (hypertension, acute myocardial infarction, atrial fibrillation, HF, and diabetes mellitus) has been observed in $>95 \%$ of patients with COPD [6]. Management of COPD is challenging due to 
the prevalence of comorbidities such as HF and risk factors such as diabetes or hypertension [7]. Comorbidities are associated with COPD exacerbation risk and increased length of stay [8].

The prevalence of comorbidities in HF is also high, with up to $40 \%$ of patients having five or more noncardiac comorbidities [9]. The proportion of HF patients with five or more chronic comorbid conditions is increasing over time, from $42 \%$ (between 1988-1994) to $58 \%$ (between 2003-2008) [10]. Similarly to COPD, comorbidities are associated with higher likelihood of readmission [11] and a significantly longer mean length of stay [12].

Our objective was to evaluate the frequency of comorbidities among patients with COPD or HF admitted to Calgary hospitals and their influence on length of stay and total patient-days in hospital. A secondary objective was to identify the most commonly occurring co-morbidities with COPD and HF.

\section{Materials And Methods \\ Data sources}

The province of Alberta in Canada publicly administers healthcare to all residents and tracks access to healthcare using a unique personalized healthcare number (PHN). All hospital discharges for individuals with a valid PHN are recorded and compiled in the Inpatient Discharge Abstract Database. This dataset includes up to 25 diagnostic codes using the International Classification of Disease 10th version (ICD-10) and provides information on hospital location as well as admission and discharge date which, allows for calculation of length of hospital stay.

\section{Study population}

The Discharge Abstract Database was used to identify all patients over the age of 18 with a valid PHN admitted to a Calgary hospital from April 1, 2017 to March 31, 2019 who had an ICD-10 diagnostic code for either COPD or HF recorded in any position in their discharge abstract. COPD was defined with ICD-10 codes J41, J42, J43, and J44 which have been validated in population-based health administrative databases and compared to chart reviews for sensitivity [13]. HF was defined with ICD-10 code I50 which has been validated with administrative data [14]. Individuals who died during the study period were included.

\section{Case ascertainment}

For our analysis, we created a 'patient profile' by assigning all diagnosis codes associated with a patient for any admission during the study period to that patient for all admissions during the study period, even if it was not coded as a diagnosis on a particular hospital admission. The rationale for a 'patient profile' is that any recently documented diagnosis is likely to have contributed to overall patient morbidity, complexity of management, and total days in hospital or readmission during the study period [15]. The primary outcome was length of hospital stay in days. The cumulative number of days in hospital over the study period for each patient profile was calculated.

Age at initial admission over the study period, sex, and comorbidities were explored as covariates. Comorbidities were defined using the Elixhauser Comorbidity Index and analyzed both as a continuous variable, and stratified as: 1) 0 comorbidities (a single diagnosis of either COPD or HF); 2) between one and five comorbidities inclusive; and 3) greater than five comorbidities. Coding algorithms to define the 31 Elixhauser comorbidity groupings using ICD-10 codes have been previously reported by Quan et al. [16]. Five comorbidities was chosen because several studies have found this to be the mean number of comorbidities among patients admitted with COPD and HF [17,18].

\section{Statistical analysis}

Analyses were performed separately for COPD and HF. Baseline characteristics were explored descriptively. Proportions were described as percentages, and continuous variables were described as means with standard deviations (SD), and medians with first and third quartiles (Q1, Q3). Wilcoxon rank-sum tests and KruskalWallis non-parametric tests were used to compare continuous variables, and chi-squared tests were used to compare proportions. Crude and adjusted (for age and sex) multilevel linear regression was performed with person as the hierarchy to explore how number of Elixhauser comorbidities as a continuous variable over the study period correlated with length of stay with results reported as coefficients and $95 \%$ confidence intervals (CI). STATA/SE 14.2 (StataCorp, Texas, USA) and Oracle SQL were used for all statistical analyses with an alpha of 0.05. Graphics were performed using Tableau and Microsoft Excel. Ethics approval was granted through the Conjoint Health Research Ethics Board at the University of Calgary (Reference number: REB19-1520).

\section{Results}

Between April 1, 2017 and March 31, 2019, the total number of distinct adult patients with COPD or HF was 5,032 and 7,197 respectively; 131 patients with COPD had no comorbidities, 3,911 had one to five comorbidities, and 990 had more than five comorbidities. 47 patients with HF had no comorbidities, 6,273 


\section{Cureus}

had one to five comorbidities, and 877 had more than five comorbidities. Baseline characteristics of the study cohort are presented in Table 1 .

\begin{tabular}{|c|c|c|}
\hline Category & COPD $(n=5,032)$ & Heart failure $(n=7,197)$ \\
\hline Age in years, median (Q1, Q3) & $74(65,82)$ & $79(68,86)$ \\
\hline \multicolumn{3}{|l|}{ Sex, (n, \%) } \\
\hline Female & 2,436 (48.4) & 3,526 (48.9) \\
\hline Male & 2,596 (51.6) & $3,671(51.1)$ \\
\hline Number of visits, median (Q1, Q3) & $1(1,2)$ & $1(1,2)$ \\
\hline Length of stay in days, median $(\mathrm{Q} 1, \mathrm{Q} 3)$ & $10(5,23.5)$ & $14(7,30)$ \\
\hline \multicolumn{3}{|l|}{ Elixhauser comorbidities, (n, \%) ${ }^{a}$} \\
\hline Congestive heart failure & 1,427 (28.4) & $7,197(100.0)$ \\
\hline Cardiac arrhythmias & $1,173(23.3)$ & 3,258 (45.3) \\
\hline Valvular disease & $191(3.8)$ & 934 (13.0) \\
\hline Peripheral vascular disorder & $160(3.2)$ & $243(3.4)$ \\
\hline Hypertension - uncomplicated & 1,741 (34.6) & 3,305 (45.9) \\
\hline Hypertension - complicated & $40(0.8)$ & $206(2.9)$ \\
\hline Chronic pulmonary disease & 5,032 (100.0) & $1,463(20.3)$ \\
\hline Pulmonary circulation disorder & $345(6.9)$ & $579(8.1)$ \\
\hline Diabetes - uncomplicated & $337(6.7)$ & $102(1.4)$ \\
\hline Diabetes - complicated & $1,014(20.2)$ & 2,679 (37.2) \\
\hline Hypothyroidism & $61(1.2)$ & $136(1.9)$ \\
\hline Fluid and electrolyte disorders & 1,082 (21.5) & 1,914 (26.6) \\
\hline Renal failure & $374(7.4)$ & 958 (13.3) \\
\hline Lymphoma & $51(1.0)$ & $78(1.1)$ \\
\hline Metastatic cancer & $233(4.4)$ & $172(2.4)$ \\
\hline Solid tumor without metastasis & $447(8.9)$ & $331(4.6)$ \\
\hline Paralysis & $39(0.8)$ & $67(0.9)$ \\
\hline Other neurologic disorders & $115(2.3)$ & $190(2.6)$ \\
\hline Liver disease & $157(3.1)$ & $247(3.4)$ \\
\hline Peptic ulcer disease excluding bleeding & $20(0.4)$ & $29(0.4)$ \\
\hline Alcohol misuse & $305(6.1)$ & $183(2.5)$ \\
\hline Drug misuse & $111(2.2)$ & $76(1.1)$ \\
\hline Psychoses & $47(0.9)$ & $35(0.5)$ \\
\hline Depression & $159(3.2)$ & $232(3.2)$ \\
\hline Deficiency anemia & $200(4.0)$ & $403(5.6)$ \\
\hline Blood loss anemia & $38(0.8)$ & $108(1.5)$ \\
\hline AIDS/HIV & $4(0.1)$ & $1(0.0)$ \\
\hline Rheumatoid arthritis/collagen vascular disorder & $71(1.4)$ & $96(1.3)$ \\
\hline Coagulopathy & $319(6.3)$ & $716(10.0)$ \\
\hline
\end{tabular}




\section{Cureus}

$\begin{array}{|lcc|}\text { Obesity } & 138(3.8) & 260(3.6) \\ \text { Weight loss } & 197(3.9) & 218(3.0) \\ \text { Mortality, }(\mathrm{n}, \%)^{\mathrm{b}} & 197(3.9) & 395(8.2)\end{array}$

\section{TABLE 1: Demographic and Elixhauser comorbidity data of patients with COPD or heart failure}

a - Elixhauser comorbidities defined by ICD-10 are reported by Quan et al. 2005 [16]

b - Mortality during study period

COPD - chronic obstructive pulmonary disease, Q1 - first quartile, Q3 - third quartile

\section{COPD}

The mean (SD) number of comorbidities was 2.7 (1.3) and 6.8 (1.1) for patients with one to five and greater than five comorbidities, respectively. The most common comorbidities for COPD patients were uncomplicated hypertension (34.6\%), congestive HF (28.4\%), arrhythmias (23.3\%), fluid and electrolyte disorders (21.5\%), and complicated diabetes (20.2\%) (Table 1).

There was a significant difference in age, number of visits, and length of stay based on number of Elixhauser comorbidities (Table 2). Patients with no comorbidities (a single diagnosis of COPD) had significantly lower median acute length of stay (median 3; IQR: 3) compared to those with one to five comorbidities (median 7; IQR: 7) and more than five comorbidities (median 10; IQR: 14) with p-value $<0.001$ (Figure 1). The number of comorbidities was significantly associated with increased length of hospital stay both as a crude estimate (coefficient 4.9; 95\% CI 4.4-5.5; $\mathrm{p}<0.001$ ) and after adjusting for age and sex (coefficient 4.9; 95\% CI 4.4-5.4; $\mathrm{p}<0.001$ ) (Table 2).

\begin{tabular}{|c|c|c|c|c|}
\hline Category & $\begin{array}{l}\text { No comorbidities }^{a} \\
(n=131)\end{array}$ & $\begin{array}{l}\text { One to five comorbidities } \\
(n=4,436)\end{array}$ & $\begin{array}{l}\text { More than five comorbidities } \\
(n=465)\end{array}$ & $\begin{array}{l}\mathrm{p}- \\
\text { value }\end{array}$ \\
\hline Sex, n (\%) & & & & 0.28 \\
\hline Female & $68(51.9)$ & $2,161(48.7)$ & 207 (44.5) & \\
\hline Male & $63(48.1)$ & 2,275 (51.3) & $258(55.5)$ & \\
\hline Age, median (Q1, Q3) & $68(61,77)$ & $74(65,82)$ & $74(67,82)$ & $<0.001$ \\
\hline $\begin{array}{l}\text { Number of visits, median } \\
\text { (Q1, Q3) }\end{array}$ & $1(1,1)$ & $1(1,2)$ & $2(1,4)$ & $<0.001$ \\
\hline $\begin{array}{l}\text { Length of stay, median (Q1, } \\
\text { Q3) }\end{array}$ & $3(2,5)$ & $7(4,13)$ & $10(6,22)$ & $<0.001$ \\
\hline Cumulative days in hospital ${ }^{\mathrm{C}}$ & 490 & 62,504 & 12,166 & $<0.001$ \\
\hline Mortality, n (\%) & $0(0.0)$ & $176(4.0)$ & 21 (4.5) & 0.055 \\
\hline
\end{tabular}

TABLE 2: Differences between stratification of diagnoses in characteristics and outcomes based on the number of Elixhauser comorbidities in COPD patients

\footnotetext{
a - No comorbidities during any hospital visit over the two year study period

b - Length of stay in hospital per admission

c - Total cumulative days in hospital for all patients over the two year study period

COPD - chronic obstructive pulmonary disease, Q1 - first quartile, Q3 - third quartile
} 


\section{Cureus}

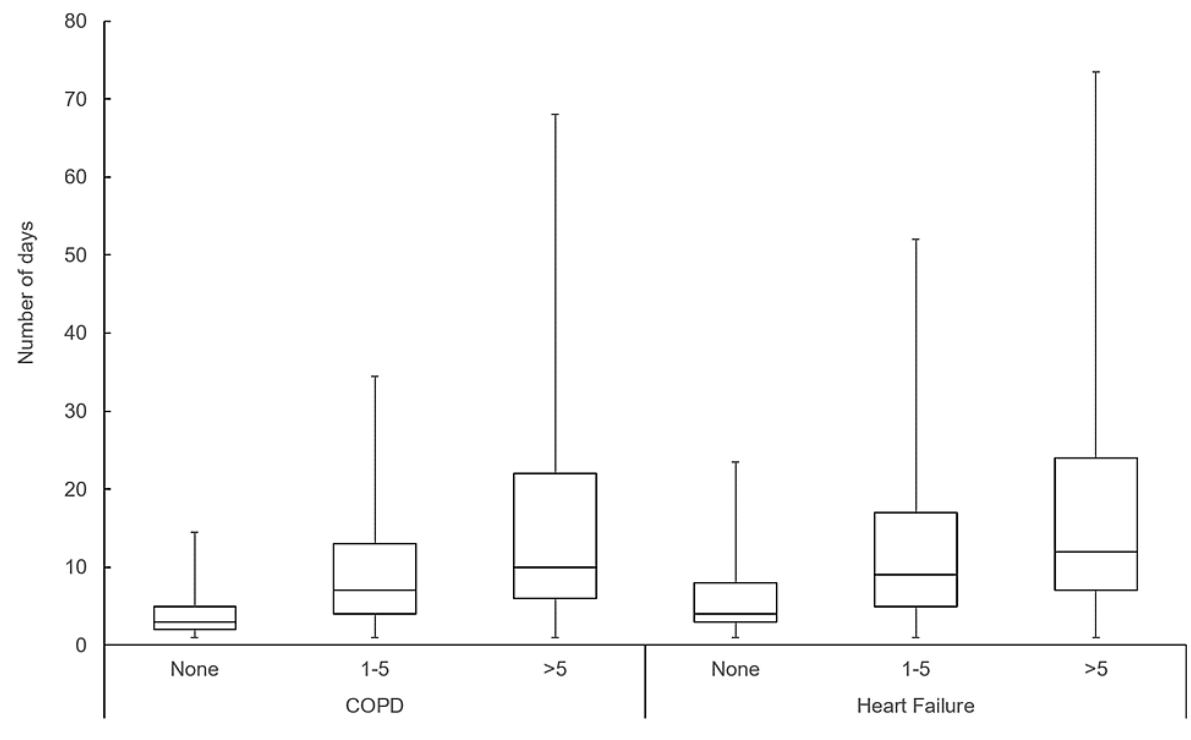

Number of comorbidities

FIGURE 1: Median length of stay stratified by the number of Elixhauser comorbidities for patients with COPD and heart failure

COPD - chronic obstructive pulmonary disease

The cumulative number of days in hospital during the study period (including acute and ALC days for all patients) was 525 for those with no comorbidities, 88,855 for those with 1-5 comorbidities, and 26,167 for those with more than five comorbidities (Figure 2).

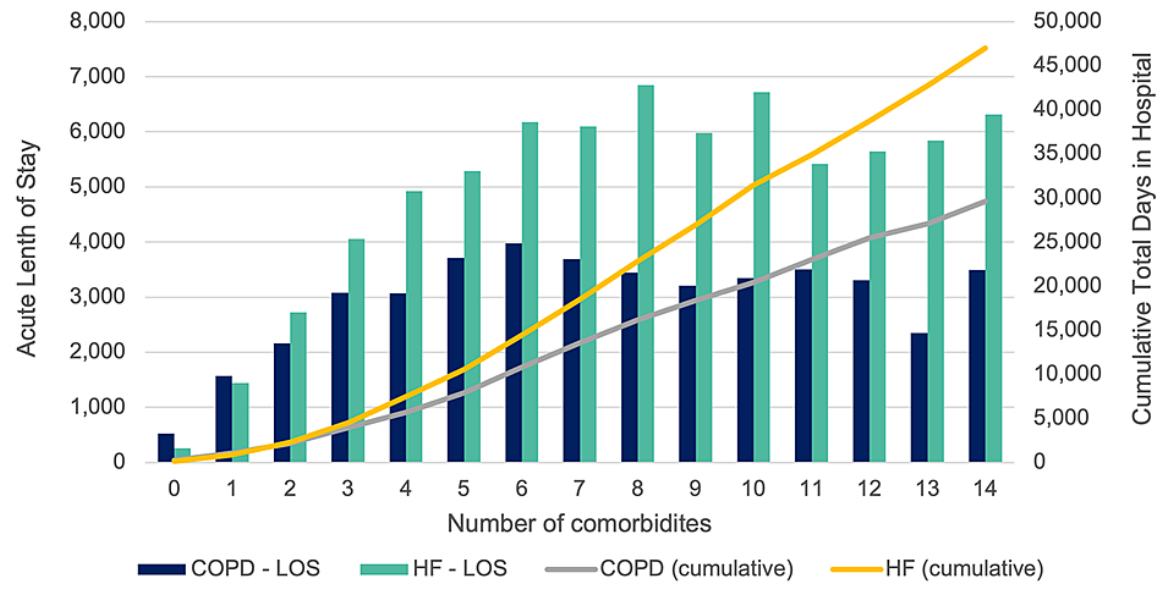

FIGURE 2: Total days in hospital and cumulative total days in hospital for all patients with COPD or HF (including acute and alternate level of care days in hospital over the two-year study period)

COPD - chronic obstructive pulmonary disease, HF - heart failure, LOS - length of stay

\section{HF}

There was a mean (SD) number of $3.2(1.2)$ and 6.6 (1.0) comorbidities in patients with one to five and greater than five comorbidities, respectively. HF patients' most common comorbidities were uncomplicated hypertension (45.9\%), arrhythmias (45.3\%), complicated diabetes (37.2\%), fluid and electrolyte disorders (26.6\%) and chronic pulmonary disease (20.3\%) (Table 1).

There was a significant difference in median length of stay for patients based on number of Elixhauser comorbidities. Patients with no comorbidities (a single diagnoses of HF) had a significantly shorter length of 
stay (median 4; IQR: 5) compared to those with one to five comorbidities (median 9; IQR: 12); and those with more than five comorbidities (median 12; IQR: 21) (p-value <0.001) (Figure 1). The number of comorbidities was significantly associated with increased length of hospital stay both as a crude estimate (coefficient 3.9; 95\% CI 3.5-4.2; $<<0.001$ ) and after adjusting for age and sex (coefficient 4.0; 95\% CI 3.6-4.3; $\mathrm{p}<0.001$ ) (Table 3).

\begin{tabular}{|c|c|c|c|c|}
\hline Category & $\begin{array}{l}\text { No comorbidities }^{a} \\
(n=47)\end{array}$ & $\begin{array}{l}\text { One to five comorbidities } \\
(n=6,273)\end{array}$ & $\begin{array}{l}\text { More than five comorbidities } \\
(\mathrm{n}=877)\end{array}$ & $\begin{array}{l}p- \\
\text { value }\end{array}$ \\
\hline Sex, n (\%) & & & & 0.205 \\
\hline Female & $23(48.9)$ & 3,098 (49.4) & 405 (46.2) & \\
\hline Male & $24(51.1)$ & $3,175(50.6)$ & $472(53.8)$ & \\
\hline Age, median (Q1, Q3) & $77(67,87)$ & $79(68,87)$ & $75(67,83)$ & $<0.001$ \\
\hline $\begin{array}{l}\text { Number of visits, median (Q1, } \\
\text { Q3) }\end{array}$ & $1(1,1)$ & $1(1,2)$ & $2(1,3)$ & $<0.001$ \\
\hline $\begin{array}{l}\text { Length of stay, median (Q1, } \\
\text { Q3) }\end{array}$ & $4(3,8)$ & $9(5,17)$ & $12(7,28)$ & $<0.001$ \\
\hline Cumulative days in hospitalc & 262 & 100,220 & 22,548 & $<0.001$ \\
\hline Mortality, n (\%) & $0(0.0)$ & $351(5.6)$ & $44(5.0)$ & 0.198 \\
\hline
\end{tabular}

\section{TABLE 3: Differences between stratification of diagnoses in characteristics and outcomes based on the number of Elixhauser comorbidities in heart failure patients}

a - No comorbidities during any hospital visit over the two-year study period

b - Length of stay in hospital per admission

c - Total cumulative days in hospital for all patients over the two-year study period

Q1 - first quartile, Q3 - third quartile

The cumulative number of days in hospital during the study period (including acute and ALC days for all patients) was 262 for those with no comorbidities, 135,555 for those with one to five comorbidities, and 47,247 for those with more than five comorbidities (Figure 2).

\section{Discussion}

In this retrospective study, we sought to determine the impact of number of comorbidities on total patientdays in hospital for patients with COPD and HF. The majority of patient-days in hospital were attributable to patients with one to five comorbidities (despite a shorter median length of stay when compared to those with more than five comorbidities). This suggests that efforts to reduce patient-days in hospital could focus on patients with one to five comorbidities, who represent 'average' patients, compared to 'outliers' who have either a single diagnosis (for whom a disease-specific order set is most appropriate) or more than five comorbidities (for whom a disease-specific order set is least appropriate). The most common co-morbidities associated with COPD and HF, including uncomplicated hypertension, arrhythmias, complicated diabetes and fluid, and electrolyte imbalances.

In the first Canadian national study to identify comorbidities with HF, hypertension, diabetes, and acute renal failure were commonly diagnosed [19]. International studies in Italy [6] and the United States [8] support the association between cardiovascular comorbidities in COPD patients and extended hospitalization. Average acute LOS at the four Calgary hospitals were comparable with national averages [1]. The similarities with our results suggest that our findings and suggestions are likely generalizable to other Canadian hospitals.

Order sets for COPD exacerbations have been associated with reductions in length of stay [20]. However, there may be unintended consequences of disease-specific order sets. Implementation of order sets for COPD and HF at other Canadian teaching hospitals had marginal gains that were partially offset by shortcomings such as order duplication and variability of order set adoption [21]. Comorbidities can influence the pathophysiologic progression of $\mathrm{HF}$ and interfere with drug and diuretic therapy options, 
Despite order sets being based on clinical practice guidelines resistance to implementation can be present due to practice inertia and lack of generalizability across practices [23]. Optimizing therapy for one condition may result in worsening of another condition [24], necessitating a more holistic approach to care (for instance, in treating acute cardiac decompensation it may be necessary to tolerate a short term worsening of renal function), and that the relative priorities of treatment may evolve over time [25] (for instance, anticoagulants may be indicated as ideal therapy but cannot be given until renal function improves).

Our results define a population of patients to prioritize in efforts to reduce total hospital utilization for all patients with HF or COPD based on the number of comorbidities. We have shown that patients admitted to Calgary hospitals with COPD or HF have a similar comorbidity profile to those in other studies. Our results suggest that co-management of the commonly occurring comorbidities in this population may be beneficial in reducing overall hospital utilization, compared with disease-specific order sets.

\section{Limitations}

Inherent in the use of administrative data is the risk for misclassification bias. We used validated codes for HF [14] and COPD [13] as well as a validated comorbidity index [26]. If misclassification did occur, it would be non-differential and bias results toward the null. An additional limitation is our assumption that each patient-day in hospital is valued in a consistent manner between conditions, patients, providers, and hospitals and constant regardless of length of admission. In reality, it is likely that individuals would attribute value to days in hospital differently depending on length of admission, severity of disease, and personal preferences [27]. Disease factors such as the type of HF or severity of disease affect hospitalization costs [28] and the fact that hospitals are often reimbursed for initial days in hospital at a higher rate than for subsequent days suggests that our assumption may not be true. Though our study was not designed to address the value of a day in hospital, future analyses could incorporate both quantitative factors such as costs as well as qualitative factors like patient quality of life based on time in hospital to better represent these issues.

Although our analysis shows that comorbidities contribute to total days in hospital, we did not investigate the impact of single-disease order sets on reducing total days in hospital for patients with HF [29] or COPD [30] (including those with comorbidities). We also did not investigate if order sets that include the management of comorbidities reduce total days in hospital. Future studies could investigate both of these issues.

\section{Conclusions}

Total days in hospital for patients with COPD or HF is highly correlated with the number of comorbidities. Patients with COPD or HF and between one to five comorbidities (compared to those with no comorbidities and those with more than five comorbidities) represent the majority of total days in hospital, and the majority of patients. Patients with COPD and HF admitted to Calgary hospitals commonly present with a 'syndrome' of related diagnoses that is similar to comorbidities identified in prior research including hypertension, arrhythmias, diabetes, and fluid and electrolyte imbalances. This highlights the importance of focusing on patients with comorbidities in efforts to reduce hospital utilization, and suggests that concurrent management of commonly occurring comorbidities for HF and COPD patients may be necessary to achieve this goal.

\section{Additional Information}

\section{Disclosures}

Human subjects: Consent was obtained or waived by all participants in this study. Conjoint Health Research Ethics Board issued approval REB19-1520. This study has been reviewed by the full Conjoint Health Research Ethics Board of the University of Calgary on October 03, 2019 . Animal subjects: All authors have confirmed that this study did not involve animal subjects or tissue. Conflicts of interest: In compliance with the ICMJE uniform disclosure form, all authors declare the following: Payment/services info: The Physician Learning Program is supported by a financial contribution from the Government of Alberta, Alberta Health - Quality and Standards. The views expressed herein do not necessarily represent the official policy of the Government of Alberta. Financial relationships: All authors have declared that they have no financial relationships at present or within the previous three years with any organizations that might have an interest in the submitted work. Other relationships: All authors have declared that there are no other relationships or activities that could appear to have influenced the submitted work.

\section{References}

1. Canadian Institute for Health Information: inpatient hospitalization, surgery and newborn statistics, 201718. (2019). Accessed: January 28, 2020: https://www.cihi.ca/en/access-data-and-reports.

2. Ezekowitz JA, O'Meara E, McDonald MA, et al.: 2017 comprehensive update of the Canadian Cardiovascular Society Guidelines for the management of heart failure. Can J Cardiol. 2017, 33:1342-433. 
10.1016/j.cjca.2017.08.022

3. Bourbeau J, Bhutani M, Hernandez P, et al.: CTS position statement: pharmacotherapy in patients with COPD-an update. Can J Respir Crit Care Sleep Med. 2017, 1:222-241. 10.1080/24745332.2017.1395588

4. Tran DT, Thanh NX, Ohinmaa A, Mayers I, Jacobs P: Current and future direct healthcare cost burden of chronic obstructive pulmonary disease in Alberta, Canada. Can J Respir Crit Care Sleep Med. 2020, 4:39-47. 10.1080/24745332.2019.1629850

5. Alberta Health Services: clinical knowledge topic: chronic obstructive pulmonary disease, adult - inpatient . (2017). Accessed: January 28, 2020: https://www.albertahealthservices.ca/cgv/Page15920.aspx.

6. Terzano C, Colamesta V, Unim B, Romani S, Meneghini A, Volpe G, La Torre G: Chronic obstructive pulmonary disease (COPD) exacerbation: impact of comorbidities on length and costs during hospitalization. Eur Rev Med Pharmacol Sci. 2017, 21:3680-3689.

7. Chen W, Thomas J, Sadatsafavi M, FitzGerald JM: Risk of cardiovascular comorbidity in patients with chronic obstructive pulmonary disease: a systematic review and meta-analysis. Lancet Respir Med. 2015, 3:631-639. 10.1016/s2213-2600(15)00241-6

8. Inabnit LS, Blanchette C, Ruban C: Comorbidities and length of stay in chronic obstructive pulmonary disease patients. Int J Chron Obstruct Pulmon Dis. 2018, 15:355-60. 10.1080/15412555.2018.1513470

9. Braunstein JB, Anderson GF, Gerstenblith G, Weller W, Niefeld M, Herbert R, Wu AW: Noncardiac comorbidity increases preventable hospitalizations and mortality among Medicare beneficiaries with chronic heart failure. J Am Coll Cardiol. 2003, 42:1226-1233. 10.1016/s0735-1097(03)00947-1

10. Wong CY, Chaudhry SI, Desai MM, Krumholz HM: Trends in comorbidity, disability, and polypharmacy in heart failure. Am J Med. 2011, 124:136-43. 10.1016/j.amjmed.2010.08.017

11. Gheorghiade M, Vaduganathan M, Fonarow GC, Bonow RO: Rehospitalization for heart failure: problems and perspectives. J Am Coll Cardiol. 2013, 61:391-403. 10.1016/j.jacc.2012.09.038

12. Carter P, Reynolds J, Carter A, Potluri S, Uppal H, Chandran S, Potluri R: The impact of psychiatric comorbidities on the length of hospital stay in patients with heart failure. Int J Cardiol. 2016, 207:292-6. 10.1016/j.ijcard.2016.01.132

13. Stein BD, Bautista A, Schumock GT, et al.: The validity of International Classification of Diseases, Ninth Revision, Clinical Modification diagnosis codes for identifying patients hospitalized for COPD exacerbations. Chest. 2012, 141:87-93. 10.1378/chest.11-0024

14. Bosco-Lévy P, Duret S, Picard F, et al.: Diagnostic accuracy of the International Classification of Diseases, Tenth Revision, codes of heart failure in an administrative database. Pharmacoepidemiol Drug Saf. 2019, 28:194-200. 10.1002/pds.4690

15. Castillo A, Edriss H, Selvan K, Nugent K: Characteristics of patients with congestive heart failure or chronic obstructive pulmonary disease readmissions within 30 days following an acute exacerbation. Qual Manag Health Care. 2017, 26:152-9. 10.1097/QMH.0000000000000143

16. Quan H, Sundararajan V, Halfon P, et al.: Coding algorithms for defining comorbidities in ICD-9-CM and ICD-10 administrative data. Med Care. 2005, 43:1130-9. 10.1097/01.mlr.0000182534.19832.83

17. Blecker S, Paul M, Taksler G, Ogedegbe G, Katz S: Heart failure-associated hospitalizations in the United States. J Am Coll Cardiol. 2013, 61:1259-67. 10.1016/j.jacc.2012.12.038

18. Divo M, Cote C, de Torres JP, et al.: Comorbidities and risk of mortality in patients with chronic obstructive pulmonary disease. Am J Respir Crit Care Med. 2012, 186:155-61. 10.1164/rccm.201201-0034OC

19. Dai S, Walsh P, Wielgosz A, Gurevich Y, Bancej C, Morrison H: Comorbidities and mortality associated with hospitalized heart failure in Canada. Can J Cardiol. 2012, 28:74-9. 10.1016/j.cjca.2011.05.002

20. Brown KE, Johnson KJ, DeRonne BM, Parenti CM, Rice KL: Order set to improve the care of patients hospitalized for an exacerbation of chronic obstructive pulmonary disease. Ann Am Thorac Soc. 2016, 13:811-5. 10.1513/AnnalsATS.201507-4660C

21. Lander B, Balka E: Balancing quality improvement and unintended effects: the impact of implementing admission order sets for chronic obstructive pulmonary disease and heart failure at two teaching hospitals. J Eval Clin Pract. 2019, 25:469-75. 10.1111/jep.13080

22. Ambrosy AP, Fonarow GC, Butler J, et al.: The global health and economic burden of hospitalizations for heart failure: lessons learned from hospitalized heart failure registries. J Am Coll Cardiol. 2014, 63:1123-33. 10.1016/j.jacc.2013.11.053

23. Cabana MD, Rand CS, Powe NR, Wu AW, Wilson MH, Abboud PA, Rubin HR: Why don't physicians follow clinical practice guidelines? A framework for improvement. JAMA. 1999, 282:1458-65. 10.1001/jama.282.15.1458

24. Tannenbaum C, Johnell K: Managing therapeutic competition in patients with heart failure, lower urinary tract symptoms and incontinence. Drugs Aging. 2014, 31:93-101. 10.1007/s40266-013-0145-1

25. Davison BA, Metra M, Senger S, et al.: Patient journey after admission for acute heart failure: length of stay, 30-day readmission and 90-day mortality. Eur J Heart Fail. 2016, 18:1041-50. 10.1002/ejhf.540

26. Austin SR, Wong YN, Uzzo RG, Beck JR, Egleston BL: Why summary comorbidity measures such as the Charlson Comorbidity Index and Elixhauser score work. Med Care. 2015, 53:e65-72. 10.1097/MLR.0b013e318297429c

27. Gualandi R, Masella C, Viglione D, Tartaglini D: Exploring the hospital patient journey: what does the patient experience?. PLoS One. 2019, 14:e0224899. 10.1371/journal.pone.0224899

28. Harjola VP, Costa S, Sund R, et al.: The type of acute heart failure and the costs of hospitalization . Int J Cardiol. 2010, 145:103-5. 10.1016/j.ijcard.2009.05.058

29. Kul S, Barbieri A, Milan E, Montag I, Vanhaecht K, Panella M: Effects of care pathways on the in-hospital treatment of heart failure: a systematic review. BMC Cardiovasc Disord. 2012, 12:81. 10.1186/1471-2261-1281

30. Lee KH, Anderson YM: The association between clinical pathways and hospital length of stay: a case study . J Med Syst. 2007, 31:79-83. 10.1007/s10916-006-9045-9 